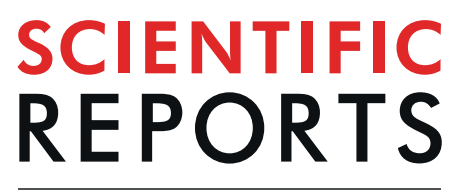

natureresearch

Check for updates

\title{
Local tissue electrical parameters predict oral mucositis in HNSCC patients: A diagnostic accuracy double-blind, randomized controlled trial
}

\author{
Gabriela Luize Guimarães Sanches ${ }^{1}$, Agna Soares da Silva Menezes ${ }^{1}$, \\ Laércio Ives Santos ${ }^{4,5}$, Cristina Paixão Durães ${ }^{1}$, Larissa Lopes Fonseca ${ }^{1}$, \\ Marcelo Perim Baldo ${ }^{3}$, Thais de Oliveira Faria ${ }^{3}$, Luciano Alves de Araújo Andrade ${ }^{6}$, \\ Petr lakovlevitch Ekel ${ }^{7}$, Sérgio Henrique Sousa Santos ${ }^{5}$, Alfredo Maurício Batista de \\ Paula $^{1}$, Lucyana Conceição Farias ${ }^{1}$, Marcos Flávio Silveira Vasconcelos D'Angelo ${ }^{2}$ \& \\ André Luiz Sena Guimarães $\mathbb{1}^{1,3,6}$ 凶
}

Oral Mucositis (OM) is a common adverse effect of head and neck squamous cell carcinoma (HNSCC) treatment. The purpose of this study was to investigate the significance of early changes in tissue electrical parameters (TEPs) in predicting the development of OM in HNSCC patients receiving radiation therapy (RT). The current study combined two study designs. The first was a case-control study. The control group comprised of RT patients who did not receive head and neck RT, and patients with HNSCC who received RT comprised the case group. In the second part of the study, the case group was included in a parallel cohort. A total of 320 patients were assessed for eligibility, and 135 patients were enrolled. Double blinding was performed, and neither the patients nor the care providers knew the measured parameters. The primary outcome was the detection of between-group changes in local TEPs over the follow-up period. The secondary outcome was the appearance of OM grades II, III, or IV and the predictive value of local TEPs in determining the incidence of OM after RT. The variables, impedance module, resistance, reactance, phase angle, and capacitance, were analyzed by the receiver operator curves (ROC). The case and control groups did not differ in demographic and clinical characteristics. Radiation therapy increased the local impedance module, resistance, reactance, and phase angle and reduced the local tissue capacitance in both groups. Evaluation of TEPs in the first week of RT correlated with the development of OM lesions during cancer therapy. ROC analysis showed that local impedance module and resistance presented higher specificity than did other parameters in predicting OM. In conclusion, local tissue electrical parameters measured at the first RT week can be useful tools to predict oral mucositis.

Therapeutic approaches for head and neck squamous cell carcinoma (HNSCC) include surgery, radiation therapy (RT), chemotherapy alone, or in combination, based on clinical staging ${ }^{1,2}$, being associated with relevant sequelae of treatment ${ }^{3}$, which negatively impact patients' lifestyle and well-being ${ }^{4-6}$. Moreover, HNSCC treatment is generally associated with high costs for patients and public health services ${ }^{7}$.

\footnotetext{
${ }^{1}$ Department of Dentistry, Universidade Estadual de Montes Claros, Minas Gerais, Montes Claros, Brazil. ${ }^{2}$ Department of Computer Science, Universidade Estadual de Montes Claros, Minas Gerais, Montes Claros, Brazil. ${ }^{3}$ Department of Pathophysiology, Universidade Estadual de Montes Claros, Montes Claros, Minas Gerais, Brazil. ${ }^{4}$ Instituto Federal do Norte de Minas Gerais, Montes Claros, Minas Gerais, Brazil. ${ }^{5}$ Institute of Agricultural Sciences, Universidade Federal de Minas Gerais (UFMG), Montes Claros, Minas Gerais, Brazil. ${ }^{6}$ Dilson Godinho Hospital, Montes Claros, Minas Gerais, Brazil. ${ }^{7}$ Pontifícia Universidade Católica de Minas Gerais, Programa de Pós-Graduação em Engenharia Elétrica, Belo Horizonte, Minas Gerais, Brazil. ${ }^{凶}$ e-mail: andreluizguimaraes@gmail.com
} 
Treatment of HNSCC has some side effects that are painful and difficult to manage ${ }^{8,9}$. Oral mucositis $(\mathrm{OM})$, dry mouth, sticky saliva, difficulty in swallowing solid foods, and loss of taste sensation are frequent complications of HNSCC RT treatment ${ }^{10}$. Oral mucositis is classified into 4 grades according to severity ${ }^{11,12}$. Grade 2 lesions are associated with pain, but the patient can still swallow solids. In grades 3 and 4 , the discomfort interferes with food intake ${ }^{10}$. Oral mucositis may necessitate modifications in the RT fraction regimen as high grades of OM need treatment interruption ${ }^{13}$. Discontinuation of RT is associated with a decrease in local control rates and reduced survival ${ }^{14,15}$. Despite the impact of OM in the patient's quality of life, there is no test to predict the incidence of OM during cancer therapy.

Many physiological processes are associated with electrical activity ${ }^{16}$, and some of these electrical parameters have proved to be useful in differentiating between normal and pathological processes ${ }^{17-21}$. TEPs are different and useful identifying tissues such as adipose and muscular tissue ${ }^{17-20}$. The majority of the studies have attempted to investigate only impedance as a marker of biological processes ${ }^{17-23}$. For example, impedance module and phase angle are widely used to evaluate body adiposity ${ }^{24}$ or, in a few cases, to detect neoplasms ${ }^{21}$.

It is known that RT alters the tissue concentrations of free radicals ${ }^{25}$, thus changing TEPs during $\mathrm{RT}^{26}$. However, no study has attempted to evaluate the local changes in impedance module, resistance, reactance, phase angle, and capacitance caused by RT. Moreover, there is no information on the association between TEPs and OM. Thus, the current study aimed to evaluate the significance of early changes in TEPs in predicting the development of OM in HNSCC patients receiving RT.

\section{Patients and methods}

Ethical approval. All performed procedures were conducted following the ethical standards of the institutional and national research committees, the 1964 Helsinki declaration, and its later amendments, or comparable ethical standards. Ethical approval for this study (Number 80731617.0.0000.5146) was obtained from the Universidade Estadual de Montes Claros Institutional Review Board. The study was also registered in the National Clinical Trials Network (UTN: U1111-1214-7398/RBR-7yygb2). Data were collected at Dilson Godinho Hospital in Brazil from March 2017 to July 2018. The trial protocol can be assessed at http://www.ensaiosclinicos.gov.br/, and ethical approval can be evaluated at http://plataformabrasil.saude.gov.br/. All patients signed the informed consent forms.

Study design. The current study followed a double-blind, prospective, diagnostic, randomized controlled cohort design ${ }^{27}$. The study was reported following the Standards for Reporting Diagnostic Accuracy (STARD) criteria $^{28}$ (Supplementary Material 1).

Sample size calculation, allocation concealment, and blinding. The sample calculation was based on previous studies ${ }^{29,30}$ and hospital statistics. The sample size calculation was performed to achieve an alpha of 0.05 , a beta of 0.05 , and a study power of 0.95 , and reach a minimal between-group difference of $50 \%$ in OM incidence. A total of 320 patients were assessed for eligibility, and 135 patients were enrolled in the study.

The study recruiter and the researcher involved with statistical analysis were not care providers. The random allocation sequence was performed not for the study recruiter. The allocation ratio was 1:1.25. Double-blinding was achieved, and neither the patients nor the care providers knew the measured parameters. The data were collected between March 2017 and July 2018 at Hospital Dilson de Quadros Godinho, located at Montes Claros city, state of Minas Gerais, Brazil.

Groups. The case group comprised of patients with HNSCC. The inclusion criteria for the case group were adults older than 18 years (both sexes) with confirmed histopathological diagnosis of squamous cell carcinoma of the base of the tongue, malignant neoplasms of other or unspecified parts of the tongue, unspecified, squamous cell carcinoma of the gum, and squamous cell carcinoma of the floor of the mouth, the palate, other or unspecified parts of the mouth, the tonsil, the oropharynx, the piriform sinus, the hypopharynx, and its variants, other or ill-defined sites in the lip, oral cavity, or pharynx receiving RT alone or in combination with chemotherapy. All patients treated with RT received 3D-RT. Patients were excluded if they had previously received head and neck $\mathrm{RT}$, presented with OM, received pacemakers or declined enrollment.

The control group included patients who received RT for prostate, urinary bladder, rectum anus, breast, and cervical cancer. The inclusion criteria for the control group were adults over 18 years of age (both sexes) with confirmed histopathological diagnosis of malignant neoplasms of the prostate, adenocarcinoma of the prostate, metastasis in other or unspecified organs of the urinary system, prostate carcinoma in situ, malignant neoplasms of the penis, unspecified, squamous cell carcinoma in situ of the mucocutaneous epithelium of the penis, other specified malignant neoplasms of the anus and anal canal, squamous cell carcinoma of the cervix uteri, adenocarcinoma of the cervix uteri, malignant neoplasms of the esophagus, unspecified, ductal carcinoma in situ of the breast, other specified malignant neoplasms of the breast and unspecified carcinoma of unspecified sites receiving RT alone or in combination with chemotherapy. All patients treated with RT received 3D-RT. Patients were excluded if they had previously received head and neck RT, presented with OM, received pacemakers or declined enrollment.

Follow-up. The case group was followed during the RT treatment (approximately 60 days). Both primary and secondary outcomes were evaluated in the case group weekly right after the start of RT treatment until the end of RT. In the case of OM occurrence, treatment was initiated following standard protocols as described elsewhere $^{11,12}$. 


\begin{tabular}{|l|}
\hline Relevant concepts \\
\hline $\begin{array}{l}\text { Impedance }(\mathrm{Z}) \text { is an expression of the opposition that a system offers } \\
\text { to alternating electric current. }\end{array}$ \\
\hline $\begin{array}{l}\text { Resistance (R) is a measure of the extent to which a substance opposes } \\
\text { the movement of electrons among its atoms. }\end{array}$ \\
\hline Reactance (X) is the amount of energy that a circuit stores ${ }^{31}$. \\
\hline $\begin{array}{l}\text { Capacitance }(\mathrm{C}) \text { is the ratio of the change in the electric charge of a } \\
\text { system to the corresponding change in its electric potential. }\end{array}$ \\
\hline
\end{tabular}

Table 1. Relevant concepts.

\begin{tabular}{|c|c|c|c|c|c|c|}
\hline & \multicolumn{2}{|l|}{ Case } & \multicolumn{2}{|c|}{ Control } & \multirow{2}{*}{\begin{tabular}{|l|} 
Total \\
$\mathbf{N}$ \\
\end{tabular}} & \multirow[b]{2}{*}{$p$-value } \\
\hline & $\mathbf{N}$ & $\%$ & $\mathrm{~N}$ & $\%$ & & \\
\hline \multicolumn{7}{|l|}{ Radiation Therapy Dose } \\
\hline$\leq 64 \mathrm{~Gy}$ & 24 & $55.8 \%$ & 19 & $44.2 \%$ & 43 & \\
\hline$>64 \mathrm{~Gy}$ & 51 & $55.4 \%$ & 41 & $44.6 \%$ & 92 & 0.97 \\
\hline \multicolumn{7}{|l|}{ Distant Metastasis (M) } \\
\hline M0 & 18 & $51.4 \%$ & 17 & $48.6 \%$ & 35 & \\
\hline M1 & 1 & $50.0 \%$ & 1 & $50.0 \%$ & 2 & \\
\hline MX & 56 & $57.1 \%$ & 42 & $42.9 \%$ & 98 & 0.83 \\
\hline \multicolumn{7}{|l|}{ Sex } \\
\hline Male & 60 & $54.5 \%$ & 50 & $45.5 \%$ & 110 & \\
\hline Female & 15 & $60.0 \%$ & 10 & $40.0 \%$ & 25 & 0.62 \\
\hline \multicolumn{7}{|l|}{ Age (years) } \\
\hline Range & $38-90$ & & $36-94$ & & & \\
\hline Mean (SD) & 63.15 & $(11.64)$ & 69.32 & $(12.94)$ & & 0.43 \\
\hline \multicolumn{7}{|l|}{ Family Income } \\
\hline$<2$ minimum wages & 70 & $55.1 \%$ & 57 & $44.9 \%$ & 127 & \\
\hline$>2$ minimum wages & 5 & $62.5 \%$ & 3 & $37.5 \%$ & 8 & 0.68 \\
\hline \multicolumn{7}{|l|}{ Schooling } \\
\hline None & 18 & $43.9 \%$ & 23 & $56.1 \%$ & 41 & \\
\hline Incomp. Elementary school & 48 & $63.2 \%$ & 28 & $36.8 \%$ & 76 & \\
\hline $\begin{array}{l}\text { Complete Elementary } \\
\text { school }\end{array}$ & 4 & $36.4 \%$ & 4 & $63.6 \%$ & 11 & \\
\hline $\begin{array}{l}\text { Complete High school/ } \\
\text { Incomplete Higher } \\
\text { Education }\end{array}$ & 5 & $71.4 \%$ & 2 & $28.6 \%$ & 7 & 0.15 \\
\hline
\end{tabular}

Table 2. Clinical characteristics of the case and control groups. Abbreviations: RT, radiation therapy. 2 Brazilian minimum wages (250 dollars). Elementary school (9 years of schooling).

Outcomes. The primary outcome was a change in local TEPs (impedance module, resistance, reactance, phase angle, and capacitance). The examination was done weekly right after the start of RT treatment until the end of RT.

The secondary outcome was the incidence of OM grades II, III, or IV, identified as previously described ${ }^{11,12}$. The presence of pain and the clinical appearance of mucositis lesions were detected during clinical examination, which was also performed weekly right after the start of RT treatment until the end of RT.

Electrical parameters. The electrical resistance properties of the tissues were evaluated based on the following variables: impedance module, resistance, reactance, phase angle, and capacitance ${ }^{20,23,24}$. Impedance $(Z)$ is an expression of the opposition that a system offers to alternating electric current ${ }^{31}$. Resistance (R) is a measure of the extent to which a substance opposes the movement of electrons among its atoms ${ }^{31}$. Reactance (X) is the amount of energy that a circuit stores ${ }^{31}$. When an alternating current passes through a body that contains reactance, the energy is released in the form of an electric field, in which case the reactance is capacitive (denoted -jX_C) ${ }^{31}$. Reactance is conventionally multiplied by the positive square root of -1 , which is the imaginary unit number called the $\mathrm{j}$ operator, to express $\mathrm{Z}$ as a complex number of the form $\mathrm{R}-\mathrm{jX} \mathrm{X} C \mathrm{C}$ (when the reactance is capacitive) ${ }^{31}$. The impedance $Z$ can also be represented in the form of impedance module $(|Z|)$ and phase angle $(\theta)$, where $|Z|=\sqrt{ }\left(R^{\wedge} 2+X^{\wedge} 2\right)$ and $\theta=\tan ^{\wedge}(-1)(X / R)^{31}$. The capacitive reactance is given by $X \_C=(1 /(2 \pi f C))$, where $f$ is the frequency of the alternating current, and $\mathrm{C}$ is the capacitance. Capacitance is the ratio of the change in the electric charge of a system to the corresponding change in its electrical potential ${ }^{31}$. The concepts are summarized in Table 1. 


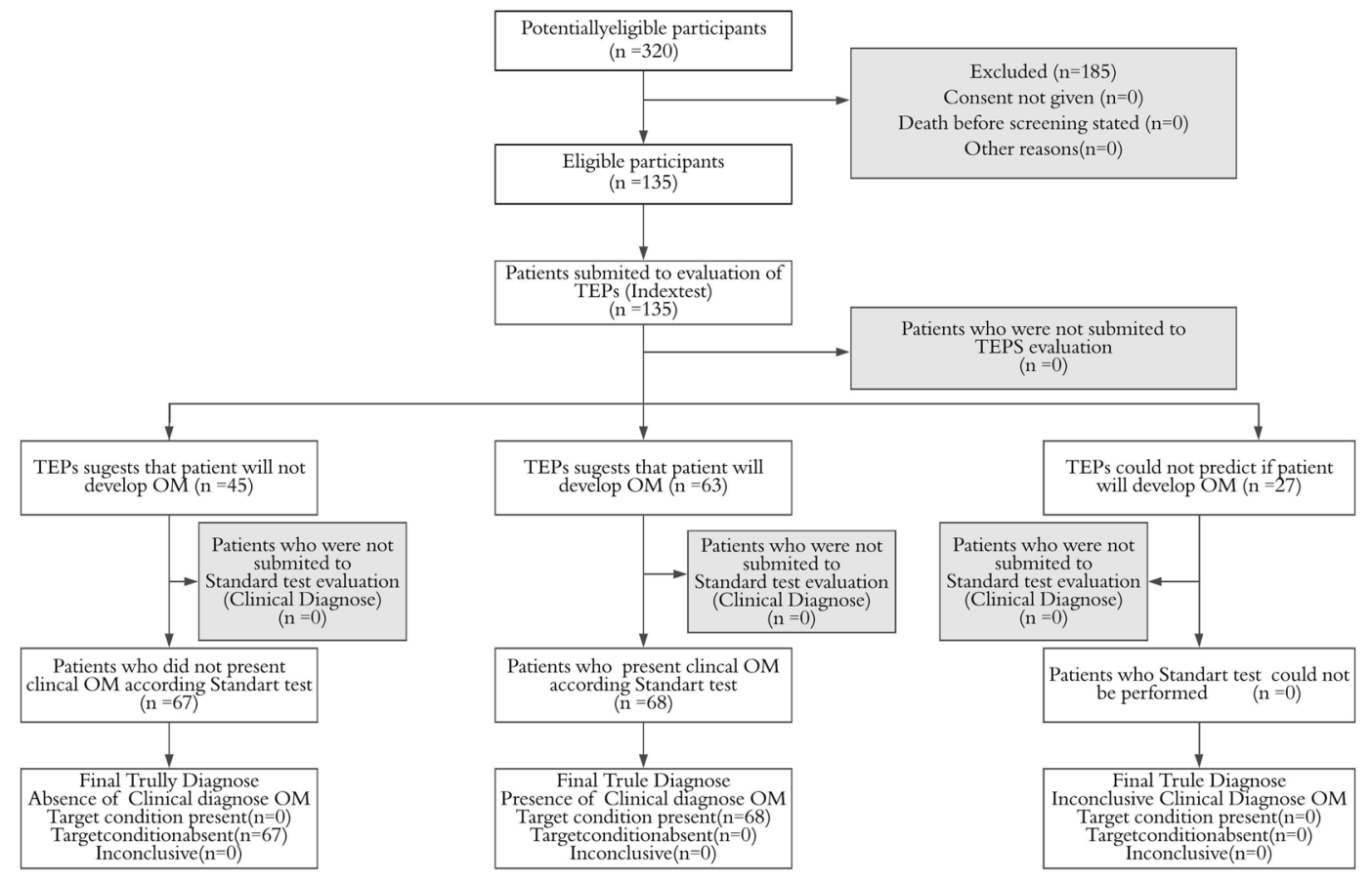

Figure 1. Flow diagram. Initially, 320 patients were potentially eligible for the study. Nevertheless, 185 did not meet the inclusion criteria. The study was performed with 135 patients. All 135 were submitted to the local and systemic TEPs evaluation, which was an index test. The standard test was the clinical diagnose of Oral Mucositis. All 135 patients were submitted to both index tests and standardized tests. The final diagnoses were the conclusion if the patient develops or not OM. According to TEPs, 48 patients will not develop mucositis. However, 67 patients did not develop OM. TEPs suggested that 63 patients will develop OM, while 68 indeed developed OM. TAPs field to conclude 27 patients.

Local TEPs (impedance module, resistance, reactance, phase angle, and capacitance) were measured in the irradiated area. Differently, systemic TEPs were collected from the whole body. Local and systemic TEPs were collected from case and control groups.

Systemic tetrapolar bioimpedance was evaluated as described elsewhere ${ }^{20,23,24}$. Briefly, a calibrated Bioelectrical Impedance Analysis Analyzer Unit (RJL Systems, Quantum BIA 101Q, Clinton Township, Michigan, EUA) using an electric current $(400 \mathrm{~mA})$ at a high frequency $(50 \mathrm{kHz})$, between 0 and $1 \mathrm{ohms}$, was used to measure bioimpedance. Electrodes (Electrodes MSGST-06, Medico Electrodes International, Uttar Pradesh, India) were placed on the right hand, wrist, foot, and ankle (Supplementary Material 2). The local tetrapolar bioimpedance was evaluated bilaterally using the ala of the nose and the tragus as reference points (Supplementary Material 2). Both local and systemic TEPs were assessed in an environment at an appropriate temperature on clean, healthy skin. The patient was held in a supine position on a nonconductive surface, arms separated from the trunk at an angle of $30^{\circ}$ and legs at $45^{\circ}$. Patients were instructed to avoid exercise, saunas, alcohol, and caffeine 8 hours before the procedure. They were advised not to eat or drink anything for one hour before the procedure, empty the bladder before the examination, and remove all metallic objects such as jewelry, rings, bracelets, and watches ${ }^{20,23,24}$. In both systemic and local bioimpedance, the wires generating the electric current were attached to the distal electrodes. Also, cables that detected voltage drop were added in the proximal. The vectors of resistance $(\mathrm{R})$ and reactance $(X c)$, were used to derive mathematical equations to determine the phase angle $\left(\theta=\tan ^{\wedge}(-1)\left(X_{c} / R\right)\right)$, impedance module $\left(Z=\sqrt{ }\left(R^{\wedge} 2+X^{\wedge} 2\right)\right)$, and capacitance $(X c=1 /((2 \pi * f * C)))$.

Oral mucositis grading. Patients were evaluated weekly, and mucositis was graded according to the World Health Organization $(\mathrm{WHO})^{12,32}$. The same dentist performed all OM gradation.

Statistical analyses. Data are shown as the mean \pm standard deviation for continuous variables and as frequency and proportion for categorical variables. To determine if the data were well-modeled by a normal distribution, the Kolmogorov-Smirnov test and the Shapiro-Wilk test were performed. These analyses revealed that the data were non-parametrically distributed. Therefore, the Mann-Whitney test was performed. Pearson correlation was performed to evaluate the changes in local and systemic tissue electric parameters.

The variables, impedance module, resistance, reactance, phase angle, and capacitance, were tested by plotting the empirical Receiver Operating Characteristic (ROC) curves using the gold standard method for comparison. The definition of areas should fill predictive indicators and classifications under the ROC curve. The Youden index was used to define the ROC cut-off values. The significance of each analysis was increased by $95 \%$. Data were applied through MedCalc Statistical Software version 16.4.3 (MedCalc Software bvba, Ostend, Belgium). A 
Local

A

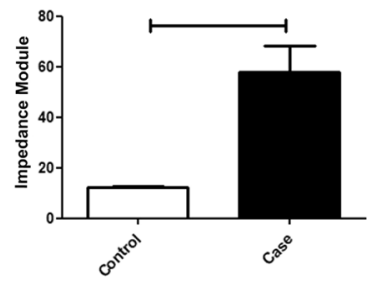

B

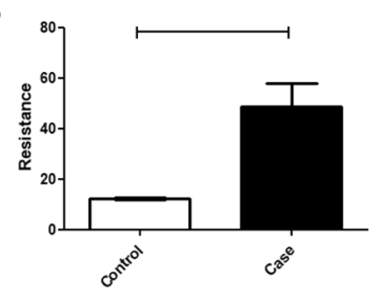

C

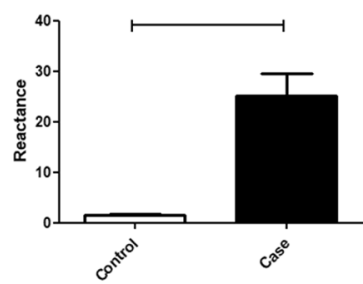

D

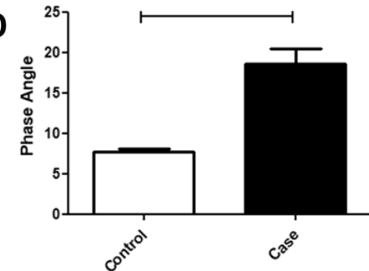

E

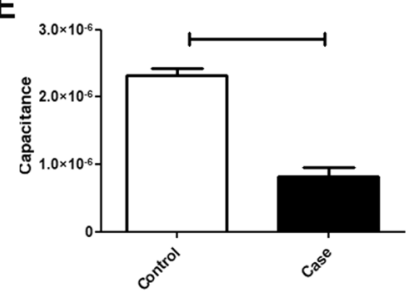

Systemic

$\mathbf{F}$

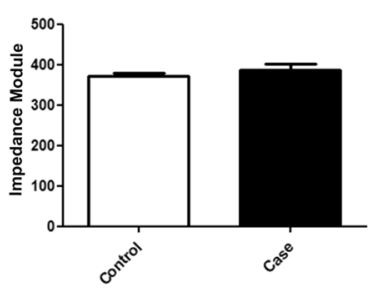

G

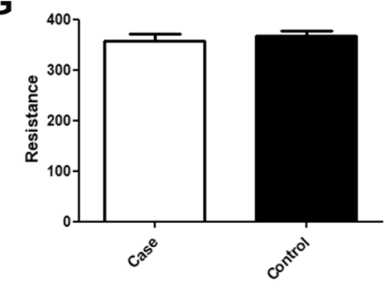

H
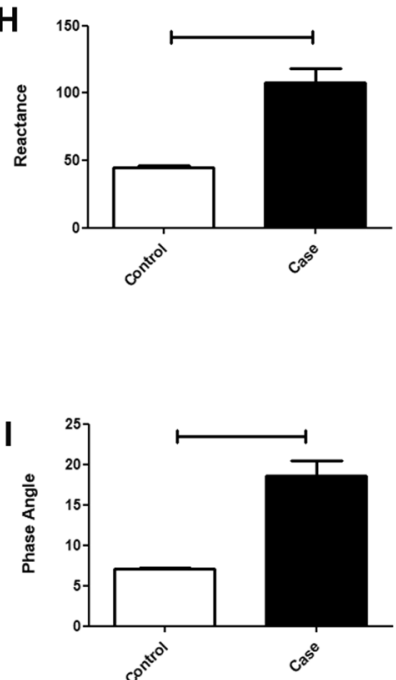

J

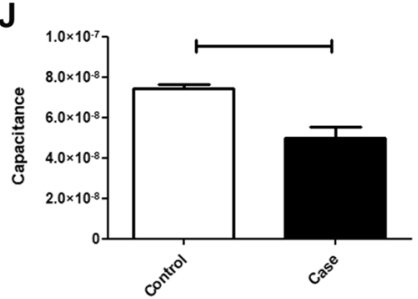

Figure 2. Systemic and local electrical parameters in case and control groups. The black bars represent the case group, while the white bars represent the control group. The bar charts depict the means; the error bars represent the standard deviation. The horizontal bars indicate significant $p$ values.

chi-square test was used for the statistical analysis of distribution differences of categorical data between groups. All statistical analyses were performed with $\mathrm{PASW}^{\circledR} \mathrm{v} 18.0$ for Windows ${ }^{\circledR}$. The results had statistical significance at $\mathrm{p}<0.05$.

Informed consent. All patients signed informed consent. 


\begin{tabular}{|c|c|c|c|c|c|}
\hline \multirow{2}{*}{$\begin{array}{l}\text { TEP } \\
\text { Impedance module }\end{array}$} & \multirow{2}{*}{\begin{tabular}{|l} 
Clinical Diagnose \\
Oral mucositis
\end{tabular}} & \multicolumn{2}{|l|}{ Cutoff } & \multirow[t]{2}{*}{\begin{tabular}{|l|}
$\begin{array}{l}\text { Pearson } \\
\text { test }\end{array}$ \\
\end{tabular}} & \multirow[t]{2}{*}{$p$-value } \\
\hline & & $\leq 13.15$ & $>13.15$ & & \\
\hline & Yes & 9 & 53 & & \\
\hline & No & 44 & 29 & 29.437 & 0.0001 \\
\hline \multirow[t]{3}{*}{ Resistance } & Oral mucositis & $\leq 13$ & $>13$ & & \\
\hline & Yes & 14 & 48 & & \\
\hline & No & 52 & 21 & 31.759 & 0.0001 \\
\hline \multirow[t]{3}{*}{ Reactance } & Oral mucositis & $\leq 4$ & $>4$ & & \\
\hline & Yes & 30 & 32 & & \\
\hline & No & 68 & 5 & 33.765 & 0.0001 \\
\hline \multirow[t]{3}{*}{ Phase angle } & Oral mucositis & $\leq 12$ & $>12$ & & \\
\hline & Yes & 27 & 35 & & \\
\hline & No & 63 & 10 & 25.576 & 0.0001 \\
\hline \multirow[t]{3}{*}{ Capacitance } & Oral mucositis & $\leq 6.37 E-07$ & $>6.37 E-07$ & & \\
\hline & Yes & 36 & 26 & & \\
\hline & No & 6 & 67 & 49.097 & 0.0001 \\
\hline \multirow[t]{5}{*}{ Impedance module } & Oral mucositis grade & $\leq 13.15$ & $>13.15$ & & \\
\hline & 0 & 44 & 23 & & \\
\hline & 2 & 8 & 49 & & \\
\hline & 3 & 1 & 9 & & \\
\hline & 4 & 0 & 1 & 39.045 & 0.0001 \\
\hline \multirow[t]{5}{*}{ Resistance } & Oral mucositis grade & $\leq 13$ & $>13$ & & \\
\hline & 0 & 52 & 15 & & \\
\hline & 2 & 13 & 44 & & \\
\hline & 3 & 1 & 9 & & \\
\hline & 4 & 0 & 1 & 44.648 & 0.0001 \\
\hline \multirow[t]{5}{*}{ Reactance } & Oral mucositis grade & $\leq 4$ & $>4$ & & \\
\hline & 0 & 65 & 2 & & \\
\hline & 2 & 27 & 30 & & \\
\hline & 3 & 6 & 4 & & \\
\hline & 4 & 0 & 1 & 41.760 & 0.0001 \\
\hline \multirow[t]{5}{*}{ Phase angle } & Oral mucositis grade & $\leq 12$ & $>12$ & & \\
\hline & 0 & 61 & 6 & & \\
\hline & 2 & 24 & 33 & & \\
\hline & 3 & 5 & 5 & & \\
\hline & 4 & 0 & 1 & 36.642 & 0.0001 \\
\hline \multirow[t]{5}{*}{ Capacitance } & Oral mucositis grade & $\leq 6.37 E-07$ & $>6.37 E-07$ &.. & \\
\hline & 0 & 37 & 30 & & \\
\hline & 2 & 5 & 52 & & \\
\hline & 3 & 0 & 10 & & \\
\hline & 4 & 0 & 1 & 50.971 & 0.0001 \\
\hline
\end{tabular}

Table 3. Correlation between tissue electrical parameters and oral mucositis. Significant $p$ values are in bold. Tissue Electrical Parameter (TEPs). All TEPs (Impedance module, Resistance, Reactance, Phase angle, Capacitance) were associated with the development of oral mucositis (OM) and the worse grade of OM.

\section{Results}

Clinical and demographic characteristics. The case group comprised 75 patients (60 men and 15 women) with a mean age of $63.15 \pm 11.64$ years. Seventy $(55.10 \%)$ patients of the case group received less than 2 Brazilian minimum wages ( 250 dollars) per month. Most patients $(\mathrm{N}=65,88 \%)$ did not complete elementary school (9 years of schooling). Regarding RT doses, $68 \%(\mathrm{~N}=51)$ of the patients from the control group received more than $64 \mathrm{~Gy}$ during the RT. In the case group $58(77.3 \%)$ patients presented OM grade 2, $8(10.7 \%)$ presented grade 3 and 3 (2.7\%) presented grade 4 (Supplementary Material 3). Taken all patients together 68 (90.6\%) OM at some point during treatment with HNSCC.

The control group comprised 60 patients ( 50 men and 10 women), slightly older than the case group, with a mean age of $69.32 \pm 12.94$ years. The family income was lower than 2 Brazilian minimum wages (250 dollars) per month for 57 (95\%) patients of the control group. Only six patients (10\%) completed elementary school. Similar to the case group, $41(68.33 \%)$ patients of the control group received more than $64 \mathrm{~Gy}$. The groups did not differ in demographic and clinical characteristics (Table 2). The flow diagram of the study is presented in Fig. 1. 


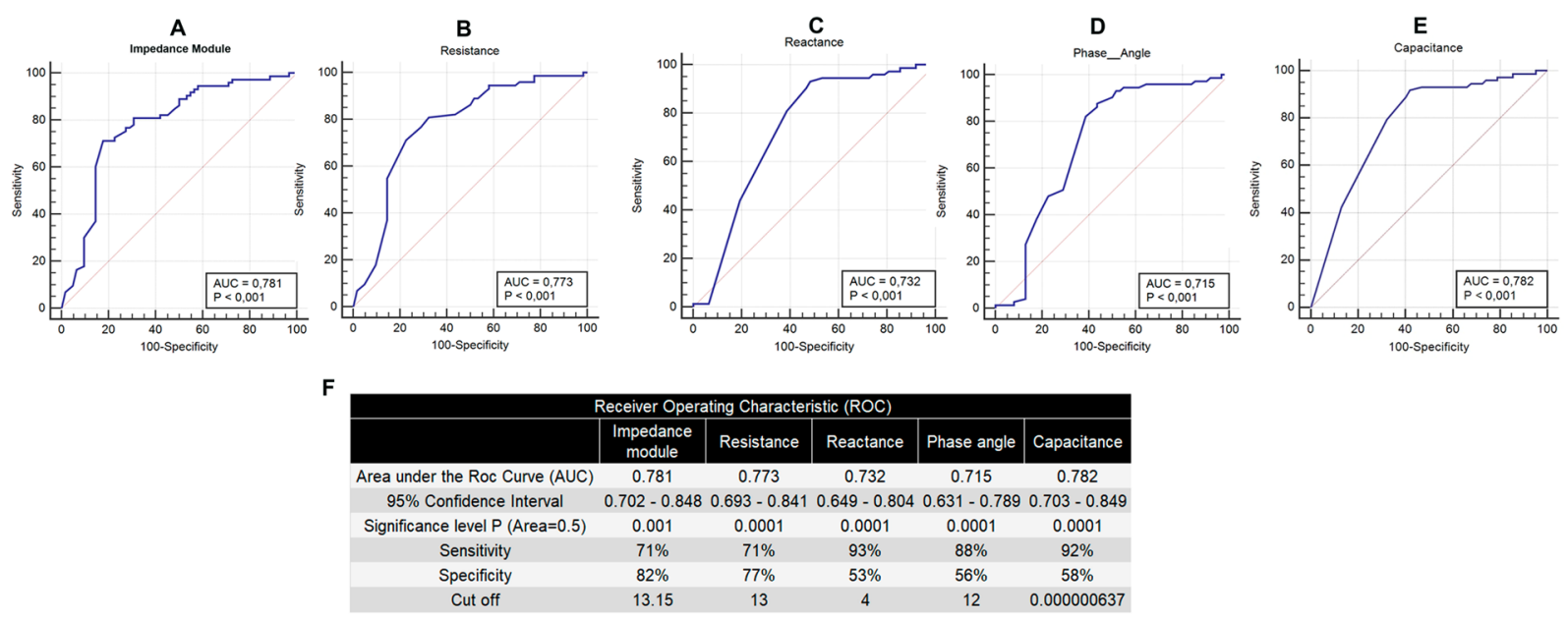

Figure 3. Receiver Operating Characteristic (ROC) curve of electrical parameters. Gold Standard by the curve for impedance module, resistance, reactance, phase angle, and capacitance (A-E, respectively).

Radiation therapy changes local tissue resistance. In the current study, only measurements of TEPs after one week of RT were used to compare with the control group. It was observed that the case group after one week of RT presented differences in all local TEPs in comparison to the control group (Fig. 2A-E). The case group presented increasing in the local impedance module (Fig. 2A), resistance (Fig. 2B), reactance (Fig. 2C), and phase angle (Fig. 2D), after one week of RT in comparison to control group. The case group also presented an increase in systemic reactance and phase angle (Fig. $2 \mathrm{H}$, I respectively) in comparison to the control group. Interestingly enough, the case group presented a reduction of both local (Fig. 2E) and systemic capacitance (Fig. 2J) in comparison to control. However, no differences between case and control groups were detected in the systemic impedance module and resistance (Fig. 2F,G).

Local tissue resistance parameters indicate oral mucositis grade. The local electrical resistance parameters were changed by RT. Moreover, the evaluation of local TEPs in the first week of RT was able to predict the development of OM grade (Table 3). The first weekly measurements of local conductive parameters were used to perform ROC analysis to define the ability of electrical parameters to predict OM and determine the cut-off of impedance module (13.15), resistance (13.00), reactance (4.00), phase angle (12.00), and capacitance $\left(6.37 \times 10^{-7}\right)$ to predict OM (Fig. 3A,B). Despite similar areas under the ROC curve, local impedance module and resistance present a better set of specificity and sensitivity than the other parameters.

\section{Discussion}

Head and neck squamous cell carcinoma, an aggressive neoplasm, poses a significant public health issue ${ }^{33}$, with developing countries showing increased incidence rates in the last year ${ }^{29}$. Treatment modalities include surgery, RT, chemotherapy ${ }^{1}$, or combination therapy, depending on the TNM classification ${ }^{1,2}$. Treatment of HNSCC is associated with sequelae such as OM, radiodermatitis, and xerostomia ${ }^{11,12,34}$. The incidence of severe OM is responsible for RT interruptions ${ }^{12}$, that may affect RT fraction regimen ${ }^{13}$, resulting in decreased local control rates and reduced survival ${ }^{14,15}$. Strategies to prevent oral mucositides include cryotherapy ${ }^{35}$, low-level laser therapy ${ }^{36}$, and two-phase intensity-modulated radiation therapy ${ }^{37}$. Currently, there is no diagnostic tool to predict OM in cancer patients ${ }^{12}$.

Similarly, there are no diagnostic tools to predict the RT side effects ${ }^{1}$ Thus, we aimed to use initial tissue electrical parameters to estimate OM in HNSCC patients receiving RT. Tissue electrical parameters are widely used to evaluate body composition, lymphedema, and obesity ${ }^{20,24,38}$. Most of the studies on tissue electrical parameters in HNSCC were performed to study body composition and used only bioimpedance ${ }^{18,22}$. Recently, a study examined an association between the status of hydration and systemic impedance with $\mathrm{OM}^{26}$. However, other tissue electrical parameters such as resistance, reactance, phase angle, and capacitance have not yet been evaluated in the literature ${ }^{18,22,26}$. In the current study, the local measure of impedance module resistance, reactance, phase angle, and capacitance changed during RT.

The ROC curve demonstrated that all local tissue electric parameters were useful to differentiate cases from control. Furthermore, the tissue electrical parameters predicted post HNSCC treatment OM. Moreover, the values of the tissue electrical parameters measured in the first week of RT predicted the appearance of OM. This suggested that local tissue electrical parameters could be useful in stratifying patients with an increased risk for OM and determining preventive strategies to avoid poor prognosis. It is essential to highlight that data of the current study suggest that the tissue electrical parameters of the first week could be used to predict future side effects of RT. Currently, there is no available tool to predict OM during cancer therapy. The current study is the first trial to demonstrate that local tissue electrical parameters can predict oral mucositis in HNSCC. Prediction of OM will identify patients needing preventive interventions or treatment. 
HNSCCs are known to contain abundant hypoxic areas ${ }^{39,41-44}$. However, studies have demonstrated that RT reduces the in vivo and in vitro levels of HIF-1 1 , miR-210, and $\mathrm{LDH}^{40-44}$. It is well-known that RT promotes ischemia-reperfusion injury ${ }^{25}$. In the current study, local impedance module, resistance, reactance, and phase angle were higher in irradiated anatomical sites. In contrast, local capacitance reduced with RT. The divergence in capacitance was expected because, by definition, impedance is directly proportional to frequency, while capacitance is inversely proportional to frequency. In corroboration with the current study, it was observed that there was an increase in the impedance parameters under ischemia and ischemia-reperfusion conditions ${ }^{19}$. Currently, there is no clinically approved tool to predict the success of $\mathrm{RT}^{1}$. In the future, the electrical parameters may also be useful to predict the effects of RT.

The main limitation of the current study was that it was conducted at a single center. Single-center studies usually present small and homogeneous patient populations. At the same time, the current study used two groups with similar clinical and demographic characteristics.

In conclusion, local tissue electrical parameters measured at the first RT week can be useful tools to predict oral mucositis.

Received: 10 October 2019; Accepted: 19 May 2020;

Published online: 12 June 2020

\section{References}

1. Mendenhall, W. M., Dagan, R., Bryant, C. M. \& Fernandes, R. P. Radiation Oncology for Head and Neck Cancer: Current Standards and Future. Changes. Oral and maxillofacial surgery clinics of North America 31, 31-38, https://doi.org/10.1016/j.coms.2018.08.003 (2019).

2. Almulla, A. et al. Radiologic-Pathologic Correlation of Extranodal Extension in Patients With Squamous Cell Carcinoma of the Oral Cavity: Implications for Future Editions of the TNM Classification. International journal of radiation oncology, biology, physics 102, 698-708, https://doi.org/10.1016/j.ijrobp.2018.05.020 (2018).

3. Langius, J. A. et al. Effect of nutritional interventions on nutritional status, quality of life and mortality in patients with head and neck cancer receiving (chemo)radiotherapy: a systematic review. Clinical nutrition 32, 671-678, https://doi.org/10.1016/j. clnu.2013.06.012 (2013).

4. De Paula, A. M. et al. Analysis of 724 cases of primary head and neck squamous cell carcinoma (HNSCC) with a focus on young patients and p53 immunolocalization. Oral oncology 45, 777-782, https://doi.org/10.1016/j.oraloncology.2008.11.015 (2009).

5. Marques-Silva, L. et al. HPV-16/18 detection does not affect the prognosis of head and neck squamous cell carcinoma in younger and older patients. Oncology letters 3, 945-949, https://doi.org/10.3892/ol.2012.588 (2012).

6. Fonseca-Silva, T. et al. Analysis of p16(CDKN2A) methylation and HPV-16 infection in oral mucosal dysplasia. Pathobiology: journal of immunopathology, molecular and cellular biology 79, 94-100, https://doi.org/10.1159/000334926 (2012).

7. Wissinger, E., Griebsch, I., Lungershausen, J., Foster, T. \& Pashos, C. L. The economic burden of head and neck cancer: a systematic literature review. PharmacoEconomics 32, 865-882, https://doi.org/10.1007/s40273-014-0169-3 (2014).

8. Vissink, A., Burlage, F. R., Spijkervet, F. K., Jansma, J. \& Coppes, R. P. Prevention and treatment of the consequences of head and neck radiotherapy. Critical reviews in oral biology and medicine: an official publication of the American Association of Oral Biologists 14, 213-225 (2003).

9. Campos, L., Simoes, A., Sa, P. H. \& Eduardo Cde, P. Improvement in quality of life of an oncological patient by laser phototherapy. Photomedicine and laser surgery 27, 371-374, https://doi.org/10.1089/pho.2008.2300 (2009).

10. Lalla, R. V. et al. Oral complications at 6 months after radiation therapy for head and neck cancer. Oral diseases 23, 1134-1143, https://doi.org/10.1111/odi.12710 (2017).

11. Soares, R. G. et al. Treatment of mucositis with combined 660-and 808-nm-wavelength low-level laser therapy reduced mucositis grade, pain, and use of analgesics: a parallel, single-blind, two-arm controlled study. Lasers in medical science, https://doi. org/10.1007/s10103-018-2549-y (2018).

12. Maria, O. M., Eliopoulos, N. \& Muanza, T. Radiation-Induced Oral Mucositis. Frontiers in oncology 7, 89, https://doi.org/10.3389/ fonc.2017.00089 (2017).

13. Biswal, B. M. Current trends in the management of oral mucositis related to cancer treatment. The Malaysian journal of medical sciences: MJMS 15, 4-13 (2008).

14. Cox, J. D. et al. Interruptions adversely affect local control and survival with hyperfractionated radiation therapy of carcinomas of the upper respiratory and digestive tracts. New evidence for accelerated proliferation from Radiation Therapy Oncology Group Protocol 8313. Cancer 69, 2744-2748 (1992).

15. Peters, L. J. et al. Critical impact of radiotherapy protocol compliance and quality in the treatment of advanced head and neck cancer: results from TROG 02.02. Journal of clinical oncology: official journal of the American Society of Clinical Oncology 28, 2996-3001, https://doi.org/10.1200/JCO.2009.27.4498 (2010).

16. Odell, M. The human body as an electric circuit. Journal of clinical forensic medicine 4, 1-6 (1997).

17. Wei, M. et al. A cell viability assessment approach based on electrical wound-healing impedance characteristics. Biosensors \& bioelectronics 124-125, 25-32, https://doi.org/10.1016/j.bios.2018.09.080 (2019).

18. Stegel, P., Kozjek, N. R., Brumen, B. A. \& Strojan, P. Bioelectrical impedance phase angle as indicator and predictor of cachexia in head and neck cancer patients treated with (chemo)radiotherapy. European journal of clinical nutrition 70, 602-606, https://doi. org/10.1038/ejcn.2016.13 (2016).

19. Pena-Mercado, E., Garcia-Lorenzana, M., Arechaga-Ocampo, E., Gonzalez-De la Rosa, C. H. \& Beltran, N. E. Evaluation of HIFlalpha and iNOS in ischemia/reperfusion gastric model: bioimpedance, histological and immunohistochemical analyses. Histology and histopathology 33, 815-823, https://doi.org/10.14670/HH-11-975 (2018).

20. Kyle, U. G. et al. Bioelectrical impedance analysis-part II: utilization in clinical practice. Clinical nutrition 23, 1430-1453, https://doi. org/10.1016/j.clnu.2004.09.012 (2004).

21. Tosi, A. L. et al. Microscopic histological characteristics of soft tissue sarcomas: analysis of tissue features and electrical resistance. Medical \& biological engineering \& computing 55, 1097-1108, https://doi.org/10.1007/s11517-016-1573-y (2017).

22. Malecka-Massalska, T., Mlak, R., Smolen, A. \& Morshed, K. Bioelectrical impedance phase angle and subjective global assessment in detecting malnutrition among newly diagnosed head and neck cancer patients. European archives of oto-rhino-laryngology: official journal of the European Federation of Oto-Rhino-Laryngological Societies 273, 1299-1305, https://doi.org/10.1007/s00405-015-36265 (2016).

23. Kyle, U. G. et al. Bioelectrical impedance analysis-part I: review of principles and methods. Clinical nutrition 23, 1226-1243, https:// doi.org/10.1016/j.clnu.2004.06.004 (2004).

24. Pena, G. D. G. et al. Interleukin-1 $\beta$ (rs1143634) polymorphism and adiposity traits in Quilombolas. Meta Gene 13, 78-84, https:// doi.org/10.1016/j.mgene.2017.05.004 (2017). 
25. Nahum, A. E. The radiobiology of hypofractionation. Clinical oncology 27, 260-269, https://doi.org/10.1016/j.clon.2015.02.001 (2015).

26. Brzozowska, A., Mlak, R., Golebiowski, P. \& Malecka-Massalska, T. Status of hydration assessed by bioelectrical impedance analysis: a valuable predictive factor for radiation-induced oral mucositis in head and neck cancer patients. Clinical \& translational oncology: official publication of the Federation of Spanish Oncology Societies and of the National Cancer Institute of Mexico, https://doi. org/10.1007/s12094-018-1963-8 (2018).

27. Rohrig, B., du Prel, J. B., Wachtlin, D. \& Blettner, M. Types of study in medical research: part 3 of a series on evaluation of scientific publications. Deutsches Arzteblatt international 106, 262-268, https://doi.org/10.3238/arztebl.2009.0262 (2009).

28. Cohen, J. F. et al. STARD 2015 guidelines for reporting diagnostic accuracy studies: explanation and elaboration. BMJ open 6 , e012799, https://doi.org/10.1136/bmjopen-2016-012799 (2016).

29. Ferlay, J. et al. Cancer incidence and mortality worldwide: sources, methods and major patterns in GLOBOCAN 2012. International journal of cancer 136, E359-386, https://doi.org/10.1002/ijc.29210 (2015).

30. Rosner, B. Fundamentals of biostatistics. (Brooks/Cole, Cengage Learning (2011).

31. Tipler, P. A. P. A. \& Mosca, G. Physics for scientists and engineers: with modern physics. 6th ed edn, (Palgrave Macmillan (2008).

32. Sonis, S. T. et al. Perspectives on cancer therapy-induced mucosal injury: pathogenesis, measurement, epidemiology, and consequences for patients. Cancer 100, 1995-2025, https://doi.org/10.1002/cncr.20162 (2004).

33. Marur, S. \& Forastiere, A. A. Head and Neck Squamous Cell Carcinoma: Update on Epidemiology, Diagnosis, and Treatment. Mayo Clinic proceedings 91, 386-396, https://doi.org/10.1016/j.mayocp.2015.12.017 (2016).

34. Speksnijder, C. M. et al. Oral function after oncological intervention in the oral cavity: a retrospective study. Journal of oral and maxillofacial surgery: official journal of the American Association of Oral and Maxillofacial Surgeons 68, 1231-1237, https://doi. org/10.1016/j.joms.2009.09.016 (2010).

35. Riley, P. et al. Interventions for preventing oral mucositis in patients with cancer receiving treatment: oral cryotherapy. The Cochrane database of systematic reviews, CD011552, https://doi.org/10.1002/14651858.CD011552.pub2 (2015).

36. Migliorati, C. et al. Systematic review of laser and other light therapy for the management of oral mucositis in cancer patients. Supportive care in cancer: official journal of the Multinational Association of Supportive Care in Cancer 21, 333-341, https://doi. org/10.1007/s00520-012-1605-6 (2013).

37. Zhang, H. H. \& D'Souza, W. D. A Treatment Planning Method for Better Management of Radiation-Induced Oral Mucositis in Locally Advanced Head and Neck Cancer. Journal of medical physics 43, 9-15, https://doi.org/10.4103/jmp.JMP_78_17 (2018).

38. Warren, A. G., Janz, B. A., Slavin, S. A. \& Borud, L. J. The use of bioimpedance analysis to evaluate lymphedema. Annals of plastic surgery 58, 541-543, https://doi.org/10.1097/01.sap.0000244977.84130.cf (2007).

39. Fraga, C. A. et al. A high HIF-1alpha expression genotype is associated with poor prognosis of upper aerodigestive tract carcinoma patients. Oral oncology 48, 130-135, https://doi.org/10.1016/j.oraloncology.2011.08.023 (2012).

40. de Souza, M. G. et al. Radiation Therapy Reduced Blood Levels of LDH, HIF-1alpha, and miR-210 in OSCC. Pathology oncology research: POR, https://doi.org/10.1007/s12253-018-0517-2 (2018).

41. Pinheiro, U. B. et al. Fuzzy clustering demonstrates that codon 72 SNP rs 1042522 of TP53 gene associated with HNSCC but not with prognoses. Tumour biology: the journal of the International Society for Oncodevelopmental Biology and Medicine 36, 9259-9265, https://doi.org/10.1007/s13277-015-3677-7 (2015).

42. Fonseca-Silva, T. et al. DNMT3B (C46359T) polymorphisms and immunoexpression of DNMT3b and DNMT1 proteins in oral lichen planus. Pathobiology: journal of immunopathology, molecular and cellular biology 79, 18-23, https://doi. org/10.1159/000330171 (2012).

43. Farias, L. C. et al. Effect of age on the association between p16CDKN2A methylation and DNMT3B polymorphism in head and neck carcinoma and patient survival. International journal of oncology 37, 167-176 (2010).

44. Domingos, P. L. et al. Leptin receptor polymorphism Gln223Arg (rs1137101) in oral squamous cell carcinoma and potentially malignant oral lesions. SpringerPlus 3, 683, https://doi.org/10.1186/2193-1801-3-683 (2014)

\section{Acknowledgements}

This study was supported by grants from the Conselho Nacional de Desenvolvimento Científico e Tecnológico (CNPq), Coordenação de Aperfeiçoamento de Pessoal de Nível Superior (CAPES) and the Fundação de Amparo a Pesquisa do Estado de Minas Gerais (FAPEMIG). Dr. Guimarães, Dr. D’Angelo, Dr. Santos, Dr. Baldo, and Dr. de Paula are research fellows of the CNPq. Dr. Farias is a research fellow of FAPEMIG. This study was supported by grants from the Conselho Nacional de Desenvolvimento Científico e Tecnológico (CNPq), Coordenação de Aperfeiçoamento de Pessoal de Nível Superior (CAPES) and the Fundação de Amparo a Pesquisa do Estado de Minas Gerais (FAPEMIG) and Hospital Dilson Godinho, Montes Claros, MG Brazil.

\section{Author contributions}

Study concepts: Gabriela Luize Guimarães Sanches, Agna Soares da Silva Menezes, Laércio Ives Santos, Marcos Flávio Silveira Vasconcelos D’Angelo and André Luiz Sena Guimarães. Study design Gabriela Luize Guimarães Sanches, Agna Soares da Silva Menezes, Laércio Ives Santos, Marcos Marcelo Perim Baldo, Thais de Oliveira Faria, Flávio Silveira Vasconcelos D’Angelo and André Luiz Sena Guimarães. Data acquisition: Gabriela Luize Guimarães Sanches, Agna Soares da Silva Menezes, Laércio Ives Santos, Cristina Paixão Durães, Larissa Lopes Fonseca, Marcelo Perim Baldo, Thais de Oliveira Faria, Luciano Alves de Araújo Andrade and Petr Iakovlevitch Ekel. Quality control of data and algorithms: Marcelo Perim Baldo, Thais de Oliveira Faria, Luciano Alves de Araújo Andrade, Petr Iakovlevitch Ekel, Sérgio Henrique Sousa Santos, Alfredo Maurício Batista de Paula, Lucyana Conceição Farias, Marcos Flávio Silveira Vasconcelos D’Angelo and André Luiz Sena Guimarães. Data analysis and interpretation, Gabriela Luize Guimarães Sanches, Agna Soares da Silva Menezes, Lucyana Conceição Farias, Marcos Flávio Silveira Vasconcelos D’Angelo and André Luiz Sena Guimarães. Statistical analysis: Marcelo Perim Baldo, Thais de Oliveira Faria, Luciano Alves de Araújo Andrade, Petr Iakovlevitch Ekel, Sérgio Henrique Sousa Santos and Alfredo Maurício Batista de Paula. Manuscript editing: Gabriela Luize Guimarães Sanches, Agna Soares da Silva Menezes, Laércio Ives Santos, Cristina Paixão Durães, Larissa Lopes Fonseca, Marcelo Perim Baldo, Thais de Oliveira Faria, Luciano Alves de Araújo Andrade, Petr Iakovlevitch Ekel and André Luiz Sena Guimarães. Manuscript review: Sérgio Henrique Sousa Santos, Alfredo Maurício Batista de Paula, Lucyana Conceição Farias, Marcos Flávio Silveira Vasconcelos D’Angelo and André Luiz Sena Guimarães. 


\section{Competing interests}

Gabriela Luize Guimarães Sanches declares that she has no conflict of interest, Agna Soares da Silva Menezes declares that she has no conflict of interest, Laércio Ives Santos, Cristina Paixão Durães declares that she has no conflict of interest, Larissa Lopes Fonseca declares that she has no conflict of interest, Marcelo Perim Baldo declares that he has no conflict of interest, Thais de Oliveira Faria declares that she has no conflict of interest, Luciano Alves de Araújo Andrade declares that he has no conflict of interest, Petr Iakovlevitch Ekel declares that he has no conflict of interest, Sérgio Henrique Sousa Santos declares that he has no conflict of interest, Alfredo Maurício Batista de Paula declares that he has no conflict of interest, Lucyana Conceição Farias declares that she has no conflict of interest, Marcos Flávio Silveira Vasconcelos D’Angelo declares that he has no conflict of interest, and André Luiz Sena Guimarães declares that he has no conflict of interest.

\section{Additional information}

Supplementary information is available for this paper at https://doi.org/10.1038/s41598-020-66351-9.

Correspondence and requests for materials should be addressed to A.L.S.G.

Reprints and permissions information is available at www.nature.com/reprints.

Publisher's note Springer Nature remains neutral with regard to jurisdictional claims in published maps and institutional affiliations.

(c) (i) Open Access This article is licensed under a Creative Commons Attribution 4.0 International License, which permits use, sharing, adaptation, distribution and reproduction in any medium or format, as long as you give appropriate credit to the original author(s) and the source, provide a link to the Creative Commons license, and indicate if changes were made. The images or other third party material in this article are included in the article's Creative Commons license, unless indicated otherwise in a credit line to the material. If material is not included in the article's Creative Commons license and your intended use is not permitted by statutory regulation or exceeds the permitted use, you will need to obtain permission directly from the copyright holder. To view a copy of this license, visit http://creativecommons.org/licenses/by/4.0/.

(C) The Author(s) 2020 